NASA/TM-2002-210728

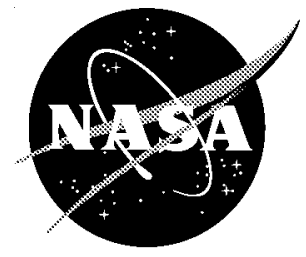

\title{
Comparison of Relative Navigation Solutions Applied Between Two Aircraft
}

Glenn Bever, Peter Urschel, and Curtis E. Hanson

NASA Dryden Flight Research Center

Edwards, California

June 2002 


\section{The NASA STI Program Office...in Profile}

Since its founding, NASA has been dedicated to the advancement of aeronautics and space science. The NASA Scientific and Technical Information (STI) Program Office plays a key part in helping NASA maintain this important role.

The NASA STI Program Office is operated by Langley Research Center, the lead center for NASA's scientific and technical information. The NASA STI Program Office provides access to the NASA STI Database, the largest collection of aeronautical and space science STI in the world. The Program Office is also NASA's institutional mechanism for disseminating the results of its research and development activities. These results are published by NASA in the NASA STI Report Series, which includes the following report types:

- TECHNICAL PUBLICATION. Reports of completed research or a major significant phase of research that present the results of NASA programs and include extensive data or theoretical analysis. Includes compilations of significant scientific and technical data and information deemed to be of continuing reference value. NASA's counterpart of peer-reviewed formal professional papers but has less stringent limitations on manuscript length and extent of graphic presentations.

- TECHNICAL MEMORANDUM. Scientific and technical findings that are preliminary or of specialized interest, e.g., quick release reports, working papers, and bibliographies that contain minimal annotation. Does not contain extensive analysis.

- CONTRACTOR REPORT. Scientific and technical findings by NASA-sponsored contractors and grantees.
- CONFERENCE PUBLICATION. Collected papers from scientific and technical conferences, symposia, seminars, or other meetings sponsored or cosponsored by NASA.

- SPECIAL PUBLICATION. Scientific, technical, or historical information from NASA programs, projects, and mission, often concerned with subjects having substantial public interest.

- TECHNICAL TRANSLATION. Englishlanguage translations of foreign scientific and technical material pertinent to NASA's mission.

Specialized services that complement the STI Program Office's diverse offerings include creating custom thesauri, building customized databases, organizing and publishing research results...even providing videos.

For more information about the NASA STI Program Office, see the following:

- Access the NASA STI Program Home Page at http://www.sti.nasa.gov

- E-mail your question via the Internet to help@sti.nasa.gov

- Fax your question to the NASA Access Help Desk at (301) 621-0134

- Telephone the NASA Access Help Desk at (301) 621-0390

- Write to:

NASA Access Help Desk

NASA Center for AeroSpace Information 7121 Standard Drive Hanover, MD 21076-1320 
NASA/TM-2002-210728

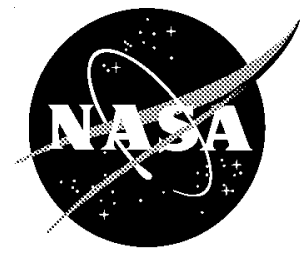

\section{Comparison of Relative Navigation Solutions Applied Between Two Aircraft}

Glenn Bever, Peter Urschel, and Curtis E. Hanson

NASA Dryden Flight Research Center

Edwards, California

National Aeronautics and

Space Administration

Dryden Flight Research Center

Edwards, California 93523-0273

June 2002 


\section{NOTICE}

Use of trade names or names of manufacturers in this document does not constitute an official endorsement of such products or manufacturers, either expressed or implied, by the National Aeronautics and Space Administration.

Available from the following:

NASA Center for AeroSpace Information (CASI)

7121 Standard Drive

Hanover, MD 21076-1320

(301) 621-0390
National Technical Information Service (NTIS) 5285 Port Royal Road Springfield, VA 22161-2171

(703) $487-4650$ 


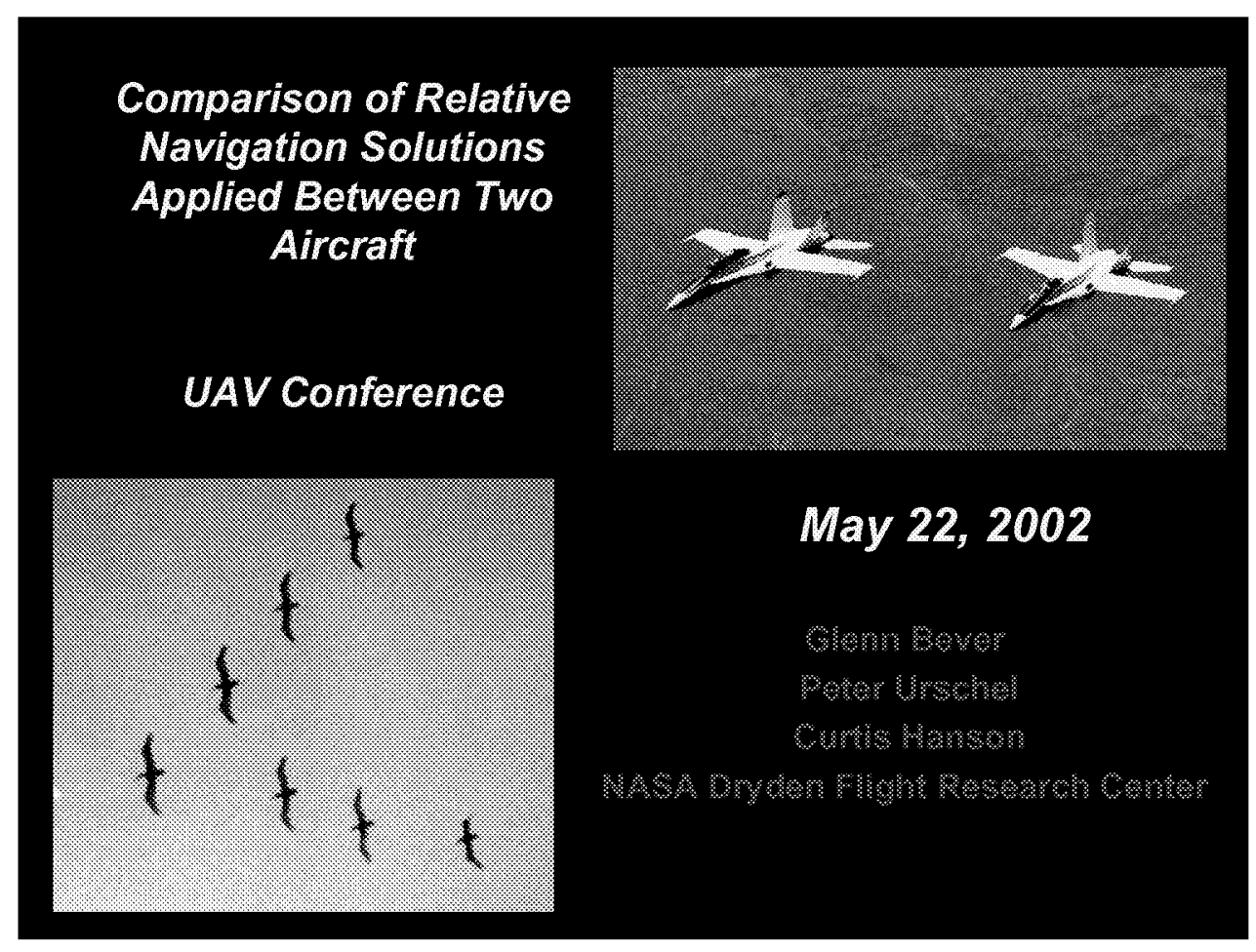

Abstract:

Use of global positioning systems (GPS) for guidance and control functions is of increasing interest to the aviation industry. Many levels of solutions exist, from the relatively simple to highly complex integrated systems.

This presentation examines three different GPS approaches to determining the relative separation between two aircraft. It presents flight test data showing the errors in each of the three approaches, tradeoffs in selecting from these three approaches, and the simplifying assumptions made for implementing applications that may reduce the requirements and therefore the cost of using them. 


\section{Outine}

- Relative Navgation Objectives

- Three Different Systems

- Characteristics of Systems

- Configuration Overview

- Agorthm Overwew

- Fllght Data: Separation Error

- Emrror Sources

- kab Data: Uncommon Satellumes

- Flight Data: Error Sources Highlighed

- Observations

- Conclusions

\section{Symbols and acronyms:}

AFF: Autonomous Formation Flight

AIMS: Airborne Information Management System

BCD: binary coded decimal

DGPS: Differential GPS

ECC: error correction code

ECEF: earth-centered earth-fixed reference frame

FFIS: Formation Flight Instrumentation System

FN: formation needles

FRF: formation reference frame

GMT: Greenwich Mean Time

GPS: global positioning system

HUD: heads up display

ILS: instrument landing system

IMU: Inertial Measurement Unit

IRIG: Inter-Range Instrumentation Group

ISMS: Independent Separation Measurement System

LAN: Local Area Network

LLA: latitude, 1ongitude, altitude

Mbit: Megabit

NED: North, East, Down reference frame
NMEA: National Marine Electronics Association

PCM: pulse code modulation (telemetry)

PC Modem: radio modem by Pacific Crest, Santa Clara, CA

PDOP: position dilution of precision

PNAV: Post Flight Navigation processing software (DGPS correction)

RS232: EIA standard

RS422: EIA standard

RT: real time

SRA: Systems Research Aircraft

UDP: user datagram protocol

$\mathrm{X}$ : distance between the leading aircraft and the trailing aircraft along the formation heading

Y: distance laterally between the two aircraft along the formation heading

$Z:$ the vertical separation between the two aircraft

Z-12 ${ }^{\mathrm{TM}}$ : GPS receiver by Ashtech, Santa Clara, CA 


\section{Relative Navigation Objectives}

Fight Test Contiguration

- Hy two arcrat at predetemined relatve positons

Relative Positioning Objectives

- provide airchat proxmity alert signal

- Display relevan airorat positioning infomation to raming aircrat pirot

- Evaluate mtegrated GPSIMU system

Relative Postitoning Data Requraments

- Display realume data in control room

- Record GPS data for postlight correction

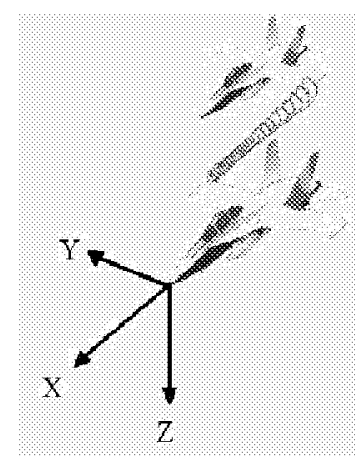

The three relative navigation approaches to be discussed were not competing systems in a flight evaluation, but were rather implemented in systems used for three different purposes. The fact that all three systems were providing information at the same time allowed a unique opportunity to compare and contrast their solutions.

The Autonomous Formation Flight (AFF) project had an overall goal to develop technologies and techniques for flying two or more aircraft in an autonomously controlled formation that could achieve greater fuel economy utilizing extra lift found in a wing vortex. Preliminary testing involved mapping the vortex behind an F-18 to determine the optimal relative positioning for such formation flight.

Flight tests were conducted to:

- evaluate a GPS-based proximity alert to the aircraft systems (for future use as an automatic autonomous flight control system disengage),

- do vortex mapping using a GPS-based pilot display, and

- evaluate an integrated GPS/inertial measurement unit (GPS/IMU) sensor that would provide information to the flight control system for autonomous control.

The data from these three systems were displayed to control room staff in real time and recorded for postflight analysis. 


\section{Three Different Systems}

- Independent Separation Measurement System (ISMS)

- simple subtraction of GPS position solutions

- time-aligned computation, but slightly delayed from real time

- Formation Needles (FN)

- same as ISMS except for:

- added filtering

- increased virtual update rate

- real-time extrapolation to remove delay

- Formation Flght instrumentation System (Fmis)

- Integration of carrier phase relative GPS with IMU

ISMS--Independent Separation Measurement System: ISMS was designed (using GPS data only) to provide a proximity alert between two aircraft and to automatically disengage an autonomous flight control system if aircraft separation is too small or closure rate is too great.

FN--Formation needles: FN provided feedback to a trailing aircraft pilot in order to allow him to position himself at a specific relative $\mathrm{Y}, \mathrm{Z}$ position with respect to a leading aircraft along the formation heading. It was designed to use GPS data and aircraft heading data. The objective was to map the wake vortex.

FFIS--Formation Flight Instrumentation System: FFIS was designed to integrate an inertial measurement unit (IMU) with carrier phase differential GPS to provide precise, reliable, relative-positioning information to a flight control system without requiring a ground DGPS station. It is, in essence, designed to be the positioning, velocity, and rate sensor to the flight control system and to be the conduit for passing such information between the aircraft flight control systems. This paper will take a cursory look at FFIS flight data as compared to ISMS and FN data.

Evaluation of the FFIS system is principally done in an AIAA paper by Walton Williamson, et. al., presented at the AIAA 1st UAV Systems, Technologies, and Operations Conference, Portsmouth, Virginia, May 2002. 


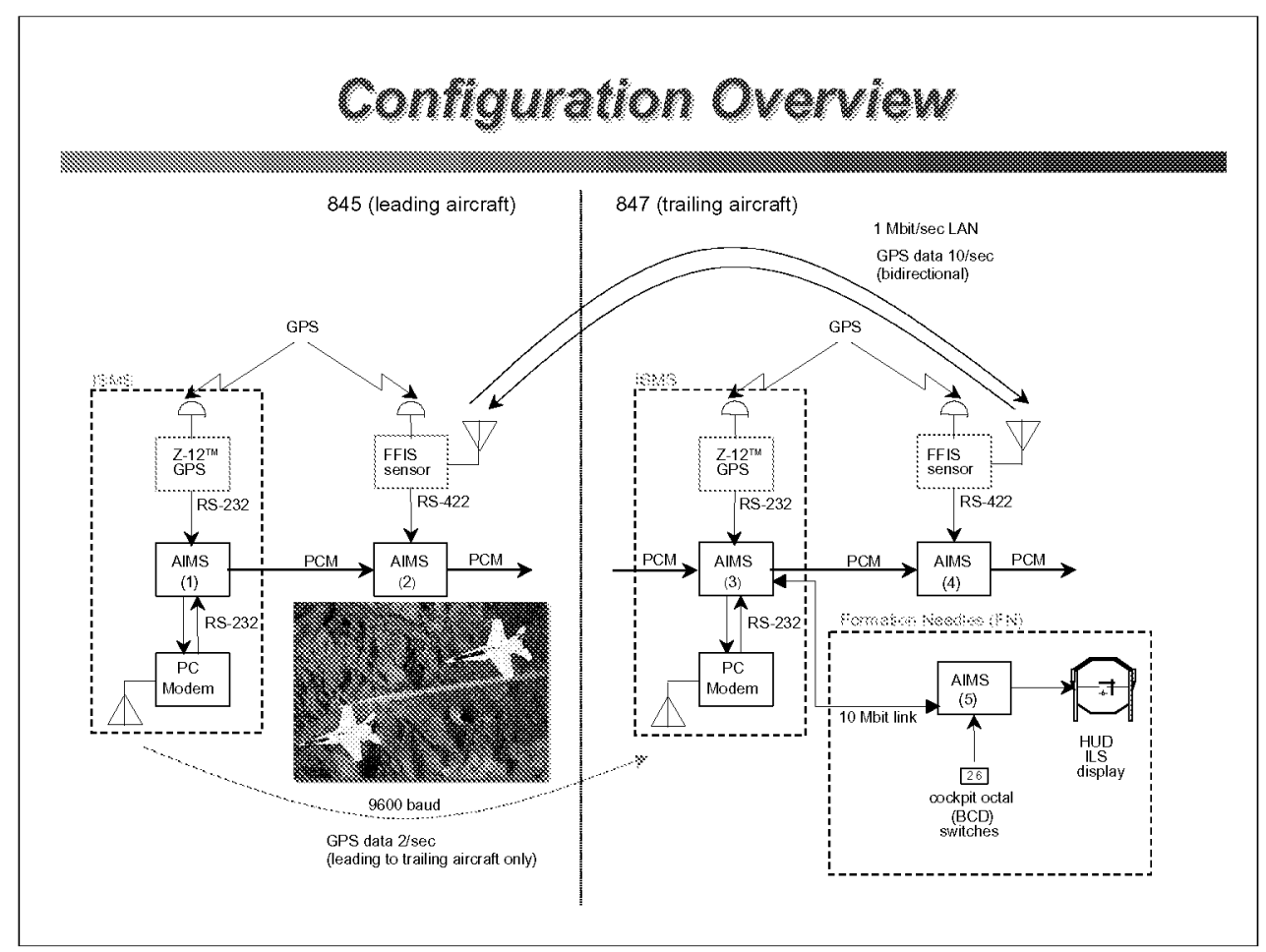

Slide 5 above, is a simplified diagram of the components in the three systems (ISMS, FN, and FFIS).

The left side represents the leading aircraft systems (F-18 tail number 845) and the right side represents the trailing aircraft systems (F-18 tail number 847). FFIS is one system and the ISMS and FN comprise the other (independent) GPS system. FN (which is on the trailing aircraft only) actually receives its data from the trailing aircraft ISMS.

The ISMS/FN radio communication uses radio modems (manufactured by Pacific Crest, Santa Clara, CA) and has a data rate of $9600 \mathrm{bits} / \mathrm{sec}$. The Pacific Crest (PC) modems use error correction coding (ECC) with 12,8 Hamming code and data interleave for burst error correction. The FFIS uses a $1 \mathrm{Mbit} / \mathrm{sec}$ Local Area Network (LAN) radio modem for user datagram protocol (UDP) communication.

The Airborne Information Management System (AIMS) is a system developed by the lead author to interface between onboard digital systems and IRIG-106 telemetry streams while also performing airborne computations in real time. For the AFF project, AIMS interfaces to GPS, pilot control/display, PC radio modem, FFIS, and telemetry systems. A total of five AIMS are used for these functions. For information regarding the AIMS system used as the core of the ISMS and FN system, reference AIAA Paper $92-4113$ by Glenn Bever, 6th Biennial Flight Test Conference, Hilton Head, SC, Aug. 24-26, 1992.

\section{ACQUISITION Process:}

- Leading aircraft AIMS (1) acquires GPS (Z-12 ${ }^{\text {TM }}$ GPS receiver by Ashtech, Santa Clara, CA) data (in NMEA 0183 format) and compresses it.

- Leading aircraft AIMS (1) sends the compressed message to the trailing aircraft through the PC Modem.

- Trailing aircraft AIMS (3) receives PC Modem data and local GPS NMEA 0183 data and computes separation in ISMS.

- Trailing aircraft AIMS (5) receives data from AIMS (3), computes formation reference frame relative positions (FN), and displays this to the trailing aircraft pilot.

- FFIS data is recorded internally and is also passed to the aircraft telemetry systems on both leading and trailing aircraft through AIMS (2) and AIMS (4), respectively. 


\section{Characteristics of Systems}

\section{- $15 x$}

- GPS receivers must select exactly the same satellites (no control)

- continues computing even if satellite mismatch noted

- uses low bandwidth communication between aircraft

- simple solution

- momaton Nedies (fry

- GPS receivers must select exactly the same satellites (no control)

- stops computing if satellite mismatch noted

- uses low bandwidth communication between aircraft

- more complex software implementation than ISMS

- Frgs

- can select common satellites (has control)

- continues computing even if all satellites lost using IMU updates

- uses high bandwidth communication between aircraft

- highly complex solution

ISMS and FN systems are dependent on the output navigation solutions of the GPS receiver. These systems cannot select what satellite sets are used in the computation. Satellite selection is determined (independently) by both GPS receivers. Having the same type of GPS receiver here is critical: the likelihood of exactly the same satellite constellation being used for navigation is increased. For these tests, both GPS receivers used were Z$12^{\mathrm{TM}}$ units by Ashtech. The communication between the ISMS on both aircraft required only 56-byte packets sent twice per second through a $9600 \mathrm{bit} / \mathrm{sec}$ radio modem.

A paper by Jennifer Hansen, et. al. at the AIAA 1st UAV Systems, Technologies, and Operations Conference provides further details regarding the vortex mapping research that used the formation needles (FN).

FFIS has full control over satellite subset selection before the navigation solution is computed. The communication between the FFIS on both aircraft required 68-byte packets wrapped in UDP packets sent ten times per second by a $1 \mathrm{Mbit} / \mathrm{sec}$ spread spectrum LAN radio modem. FFIS is still in development so during the flight test it was not very robust--which means it often lost carrier phase lock and had problems in integrating its solution with IMU data. 


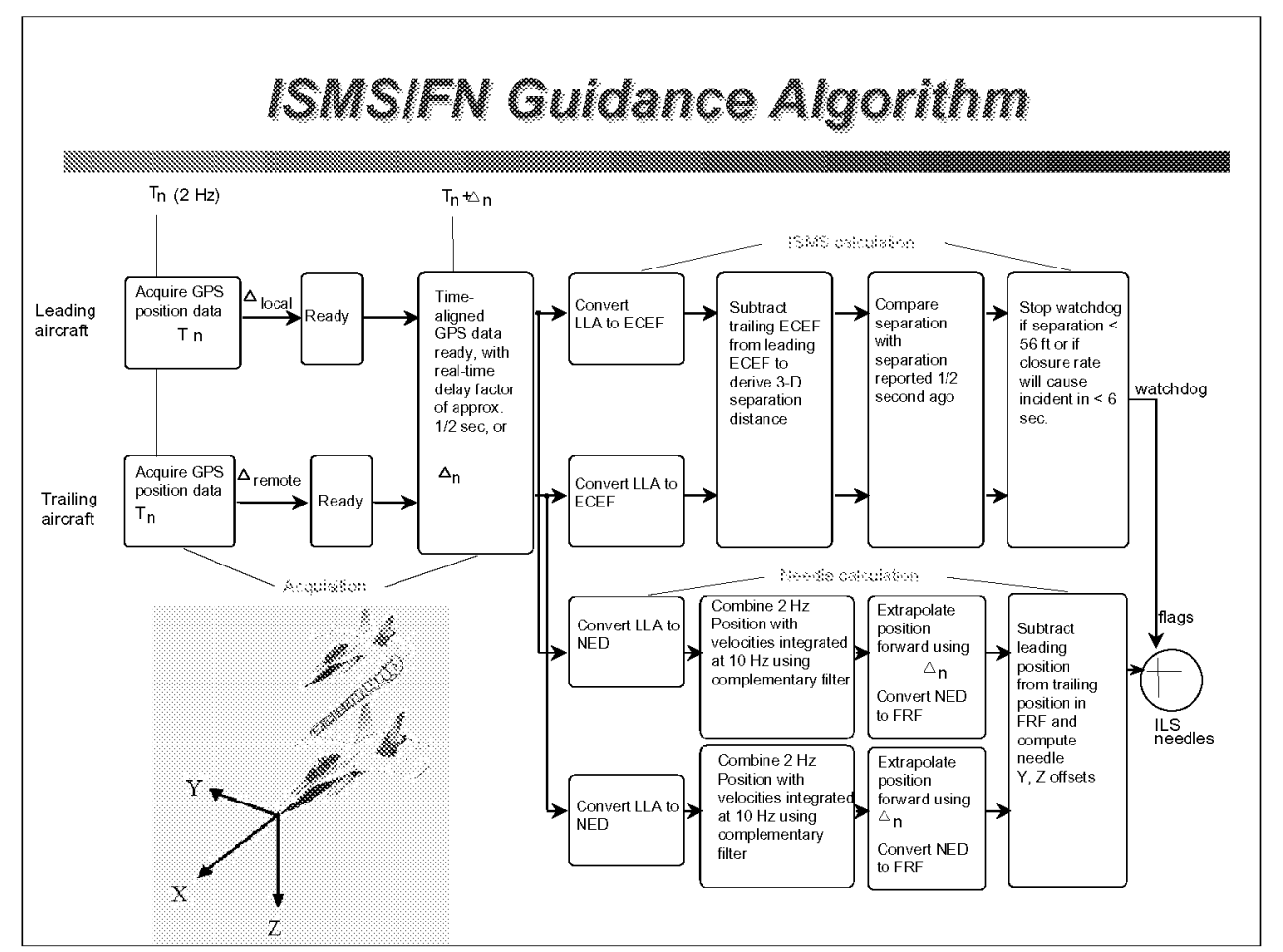

\section{ISMS calculation:}

The ISMS algorithm computes the earth-centered earth-fixed (ECEF) position of each aircraft based on received latitude, longitude, altitude (LLA) coordinates. It then calculates the difference between the leading and trailing aircraft ECEF positions and computes the scalar separation between them. The closure rate between the two aircraft is computed based on the current and previously read scalar separations.

The signal to disengage the autonomous flight control system is the stopping of a so-called 'watchdog' clock signal. For the purposes of these tests, it merely indicates to the instrumentation system that the disengage request has occurred.

If the scalar separation is less than $112 \mathrm{ft}$ (nose-to-nose), the 'watchdog' clock is stopped. (112 ft - $56 \mathrm{ft}$ aircraft length $=56 \mathrm{ft}$ nose-to-tail). The 'watchdog' clock is also stopped if the closure rate predicts a separation of $0 \mathrm{ft}$ nose-to-nose in 6 seconds.

\section{Needle (FN) calculation:}

The FN algorithm computes North, East, Down (NED) position and velocities of each aircraft relative to a fixed ground point. A complementary filter is used to integrate position with velocity to extrapolate $2 \mathrm{~Hz}$ data forward in increments of 0.1 seconds (for smoother pilot needle display). To minimize latency, data is then extrapolated forward using the measured delta time value and computed velocity vectors.

The NED coordinates are rotated into the formation heading to become formation reference frame (FRF) data, where $\mathrm{X}$ is the distance between the leading and the trailing aircraft along the formation heading, $\mathrm{Y}$ is the distance laterally between the aircraft along that heading, and $\mathrm{Z}$ is the vertical separation between the aircraft.

The computed relative position between the two aircraft (in FRF coordinates) is subtracted from the commanded relative position between the two aircraft (also in FRF) to derive the trailing aircraft offset from the desired position. This $\mathrm{Y}, \mathrm{Z}$ offset is displayed on the pilot needles. 


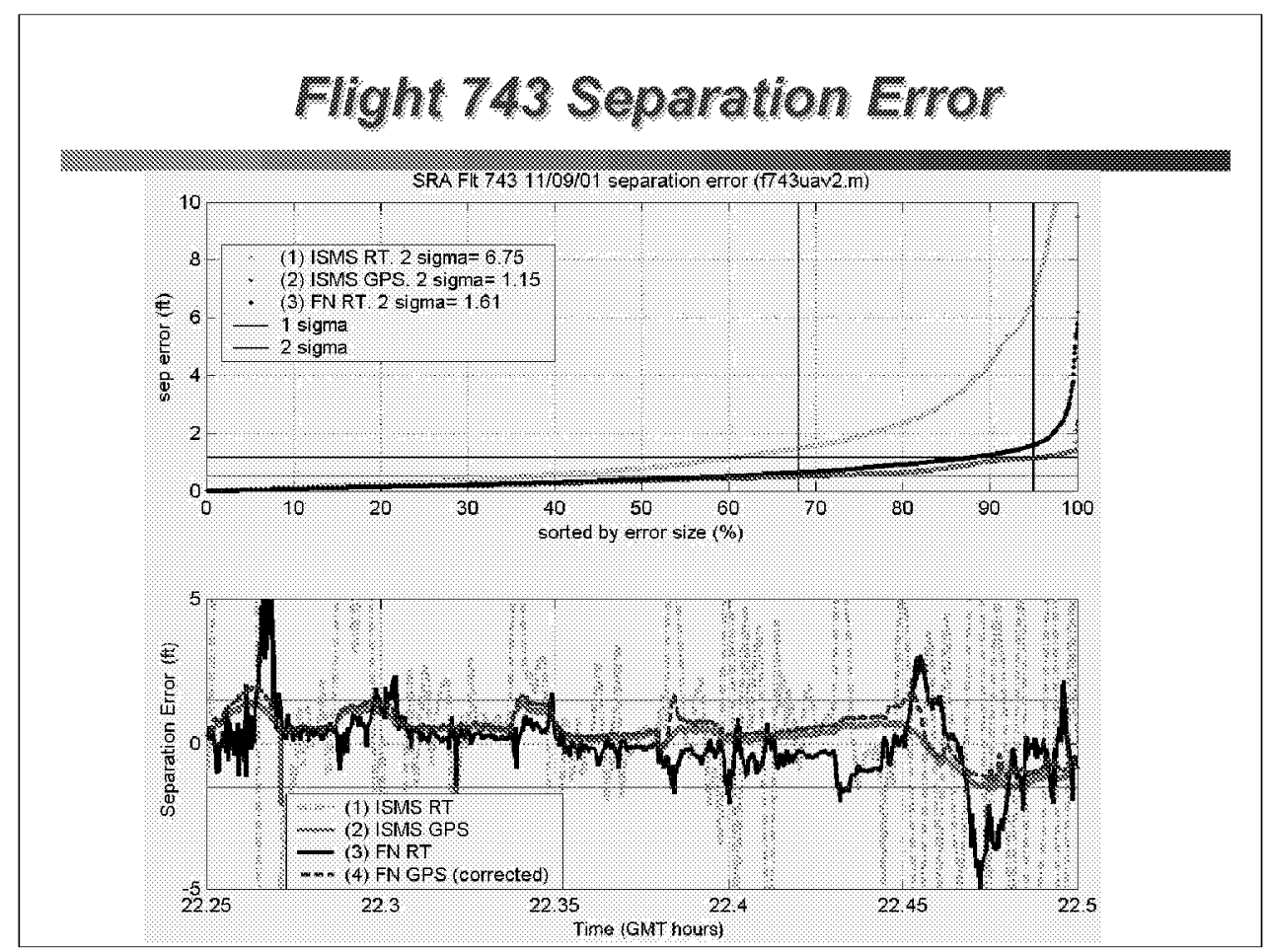

The top plot on the slide above graphically shows the computation of 1- and 2- sigma values for three of the four plots in the bottom graph. A 1-sigma error indicates that 68 percent of the data falls below this line. A 2-sigma error indicates that 95 percent of the data falls below this line. The top plot represents data from the whole flight, except for some data that was removed because of problems such as: aircraft known to be out of relative range, GPS satellite mismatch, or telemetry dropout conditions. The remaining data was sorted from smallest to largest, normalized to 100 percent of the points, and plotted. These conditions will be discussed later.

The bottom plot shows a section of a typical vortex mapping flight. It overlays the separation errors of four calculation methods on one plot. Note that it is a portion of the flight, and does not represent all of the data used in calculating the sigma error values in the top plot.

Line (1) is the ISMS error in real time (RT) (as a function of PCM RT). The error is computed by subtracting the ISMS RT position from the postflight navigation (PNAV) DGPS-corrected data. The plotted scale is only $\pm 5 \mathrm{ft}$, but it oscillates back and forth substantially in this region. This is because the data, while time-aligned between the two aircraft, is delayed by about 0.5 seconds before it is available to the PCM stream, where it is time tagged. This skews the data with respect to the GPS time-tagged PNAV data.

Line (2) is this same ISMS data as line (1) except it is plotted as a function of the GPS time in which the position data was good. This removes all latencies.

Line (3) represents the FN calculation error in real time (as a function of PCM RT). The FN algorithm computed onboard the aircraft attempts to remove the latency between the time in which the data was acquired and the real time in which it is to be used by the pilot display. You can see that it is a bit ragged, as the latency calculation is not perfect. It is fundamentally based on the same acquired data as both lines (1) and (2).

Line (4) takes the same real-time, non-DGPS-corrected data as line (3) but applies a new algorithm to it (a socalled 'corrected' algorithm). The reason for the new algorithm will be discussed on the next slide. Note that it tracks line (2) closely, except during course or altitude changes, when the predictions about flightpath will be in error. The reason that it is not as ragged as line (3) is that it plots as a function of acquired GPS time and not extrapolated real time. 


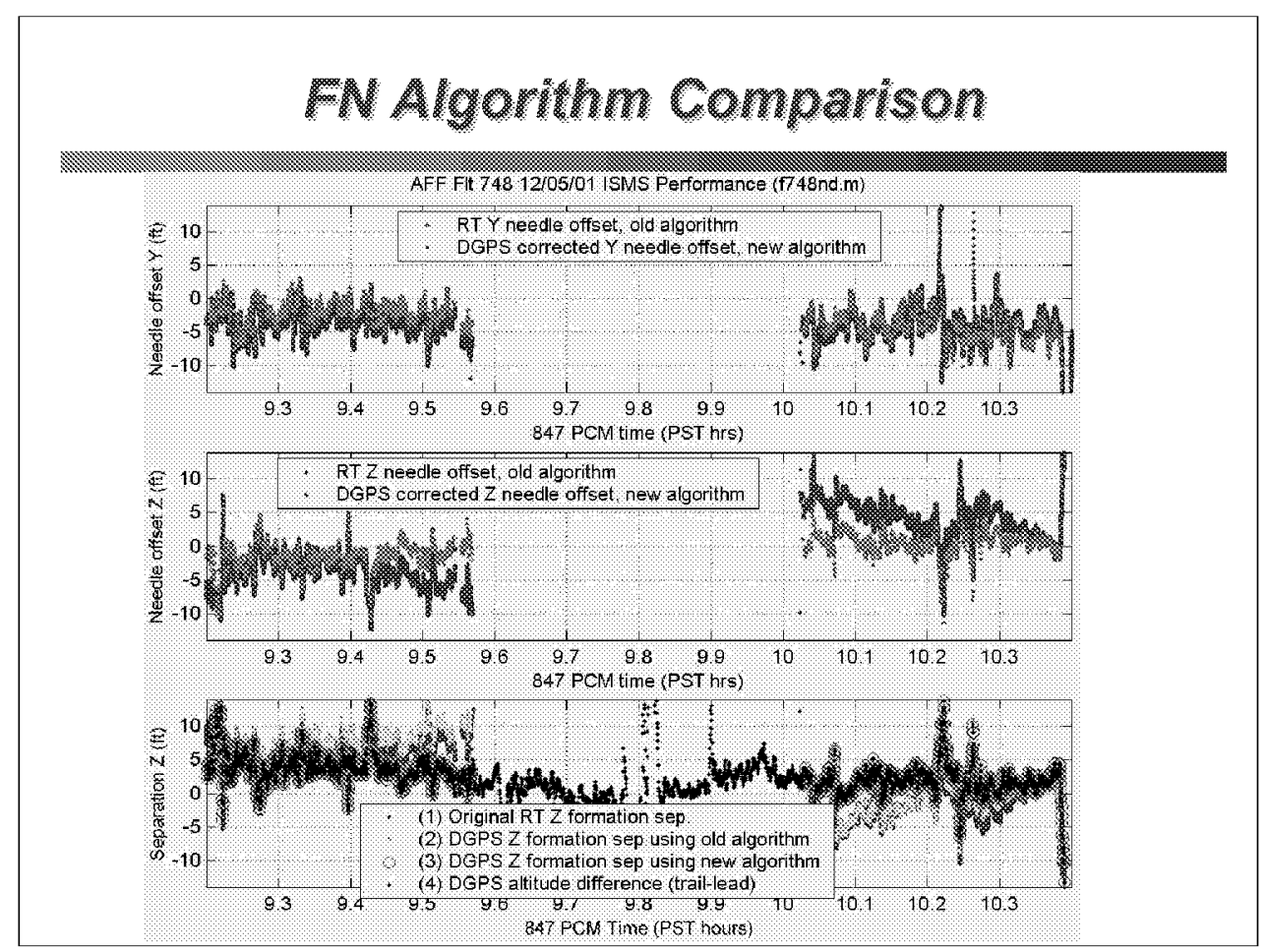

It was discovered after the flight program completed that there was a problem in the choice of algorithm used for the FN calculation. The flight data was run through a new algorithm postflight. It is 'corrected' in the sense that it uses the same real-time, non-DGPS-corrected data as the onboard algorithm, but it runs through an improved algorithm in postflight (but without using the complementary filter and velocity estimates used onboard).

The plot above illustrates the issue. On flight 748 , the whole flight was flown with the trailing pilot keeping the same station with respect to the leading aircraft. After selecting what, through experience, he recognized as the optimal station, he cross referenced the FN display. He reported that it was indicating five ft low. After about one-half hour of flight, the aircraft flew a reciprocal heading while keeping the same station. The pilot then reported the FN display was indicating five $\mathrm{ft}$ high.

Video tape from the heads up display (HUD) confirmed the pilot's assertion that the relative position of the two aircraft had not changed. A new algorithm was applied to flight data and this algorithm is plotted on this slide with the onboard computed data.

Note that the $\mathrm{Y}$, or lateral, needle saw no significant change between the two algorithms (top plot), but the Z, or vertical, needle flattened out to indicate zero for both the outbound and inbound portions of the flight (middle plot). The gap in the middle is a result of the aircraft telemetry being out of range of the acquisition site.

The bottom plot is a sanity check. As the top two plots compared non-DGPS-corrected, old-algorithm data to DGPS-corrected, new-algorithm data, there were two variables changed. In the bottom plot, Line (1) is the onboard real time (RT) aircraft Z separation data using the old algorithm. Line (2) is the DGPS-corrected data also using the onboard (old) algorithm. Lines (1) and (2) match each other closely, but neither match the pilot and HUD observations. This helps to verify that the difference between the lines in the middle plot is more a result of the algorithm difference than because one line is DGPS-corrected and the other is not. Line (3) is DGPS-corrected data using the new (corrected) algorithm. Line (4) is a straight differencing of the DGPScorrected altitudes (which is the same as line (3) if the formation reference frame algorithm was done correctly). As the line (4) data was recorded onboard, there is no gap in its data.

These plots confirm that the new algorithm is a better match to pilot and HUD video observation for flight 748 than the old one. 


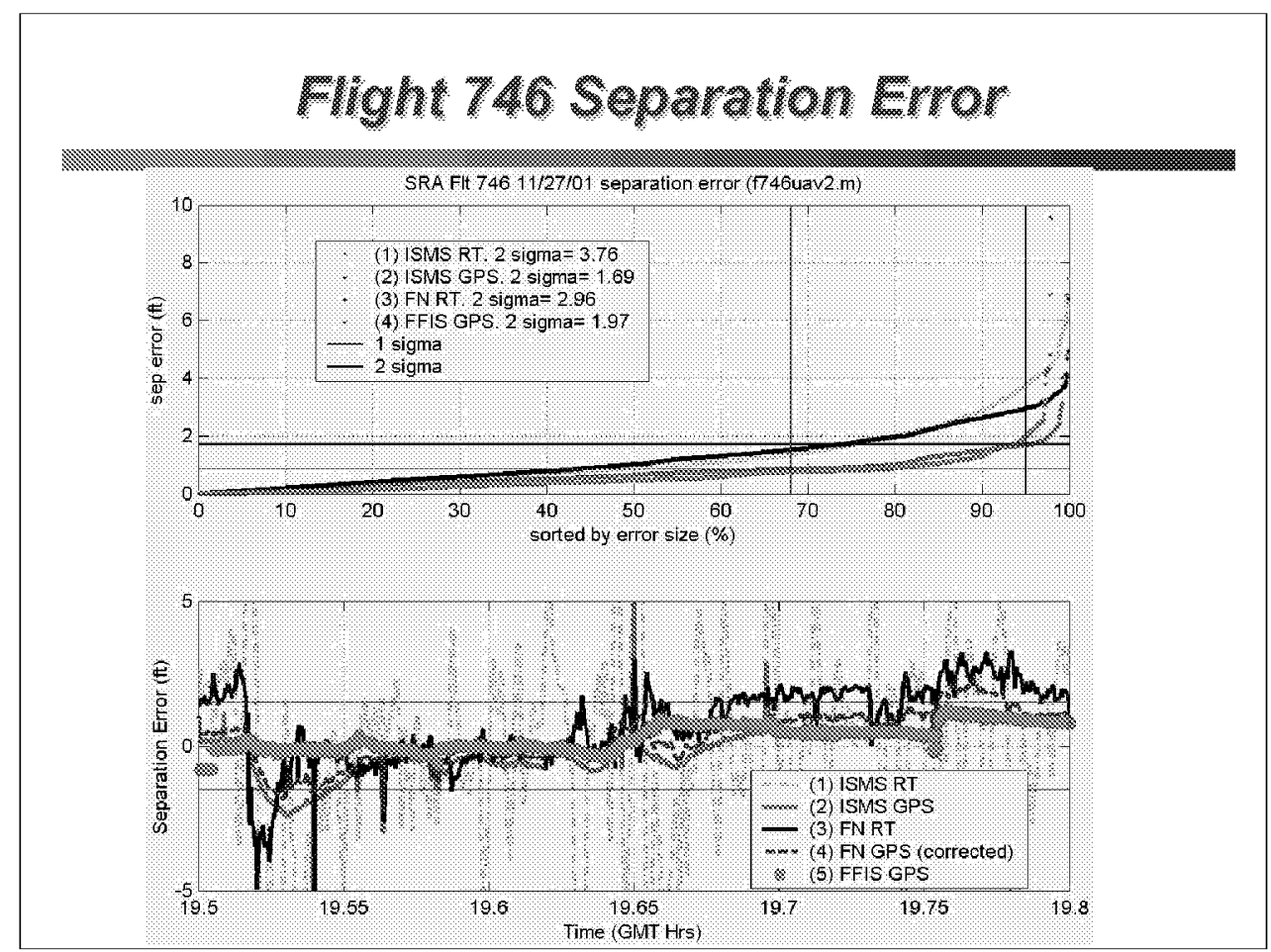

This slide represents another flight, plotted the same way as the last separation error plot, except that there is an additional plot for the FFIS error. This flight represented the longest time that the FFIS was locked in carrier phase mode (operating at maximum accuracy) so it is the best flight in which to compare FFIS error with the other methods.

The FFIS error is line (5) on the bottom plot. It is plotted against the GPS time at which the FFIS separation data was acquired--so its latencies have been removed. The FFIS data plotted here is based on carrier phase differential GPS only, the so-called 'Wald' test output, and is not blended with IMU data.

The top sigma plot illustrates that the ISMS and FFIS error distribution is almost identical for this flight. Both report a 2-sigma error of under $2 \mathrm{ft}$. The FN and real time ISMS were about 3 and $4 \mathrm{ft}$, respectively. The realtime ISMS (ISMS plotted as a function of real time) is always 'noisy' because of the latency problem--although the oscillations are not very large, and the 2-sigma error stays under $4 \mathrm{ft}$. 


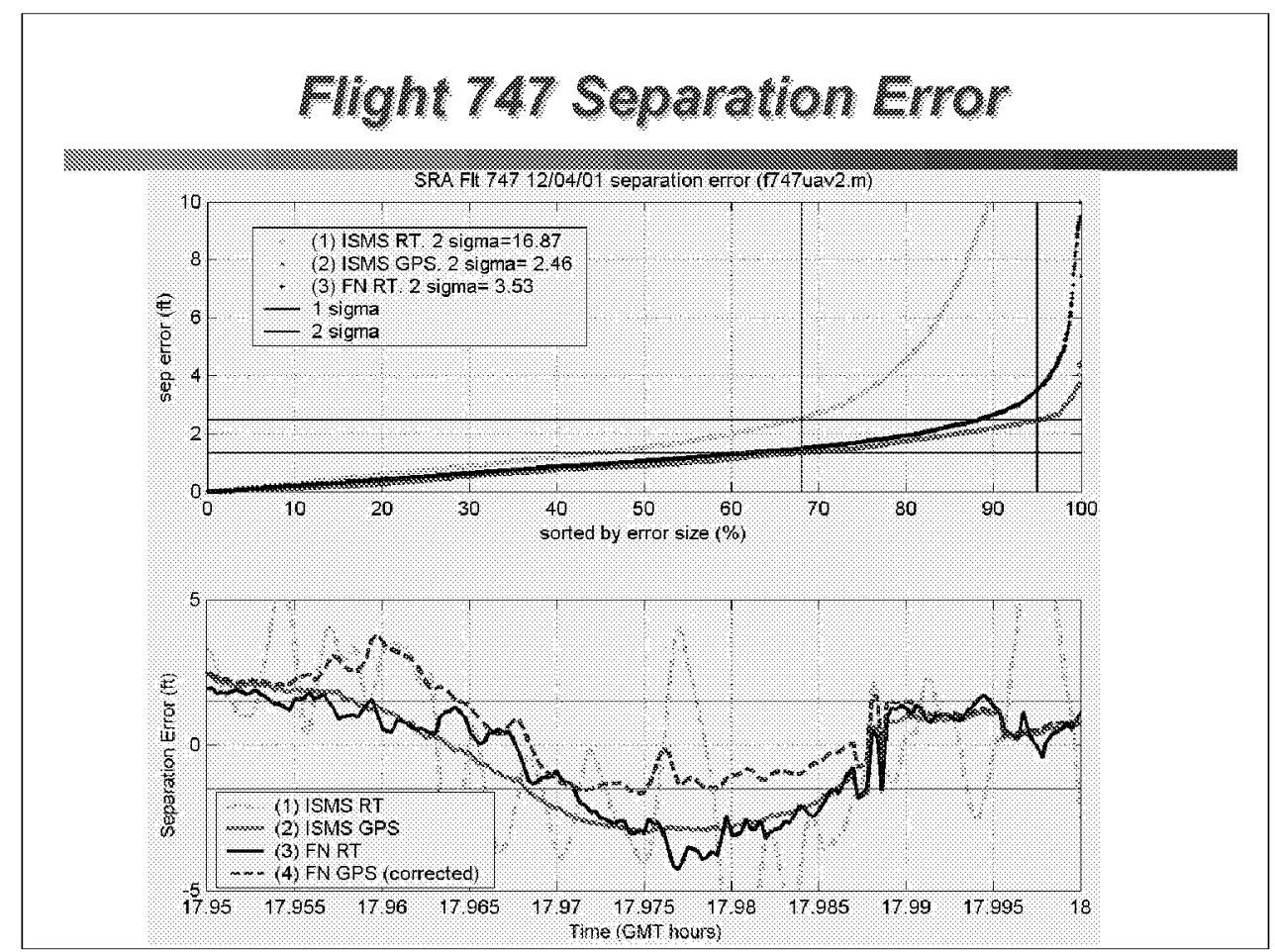

The bottom plot shows a segment where the aircraft were changing course. During the turn you can see that the error changes, but not substantially. (A more sudden turn would probably change the satellites being tracked as well as cause problems for the extrapolation of position.)

The ISMS RT 2-sigma [line (1) of the top plot] is high as a result of significant accelerations and decelerations between the two aircraft at points throughout the flight. ISMS RT error 'spikes' greater than $20 \mathrm{ft}$ (not shown here) correlate very well with relative aircraft acceleration and deceleration. If the aircraft are significantly accelerating or decelerating with respect to each other, latency is an increased problem--and the latency is about one-half second. Latency problems would be minimized if the relative separation between the two aircraft remained constant.

This acceleration and deceleration error is seen in FN RT as well, but it is not as pronounced because that algorithm has a latency compensation. 


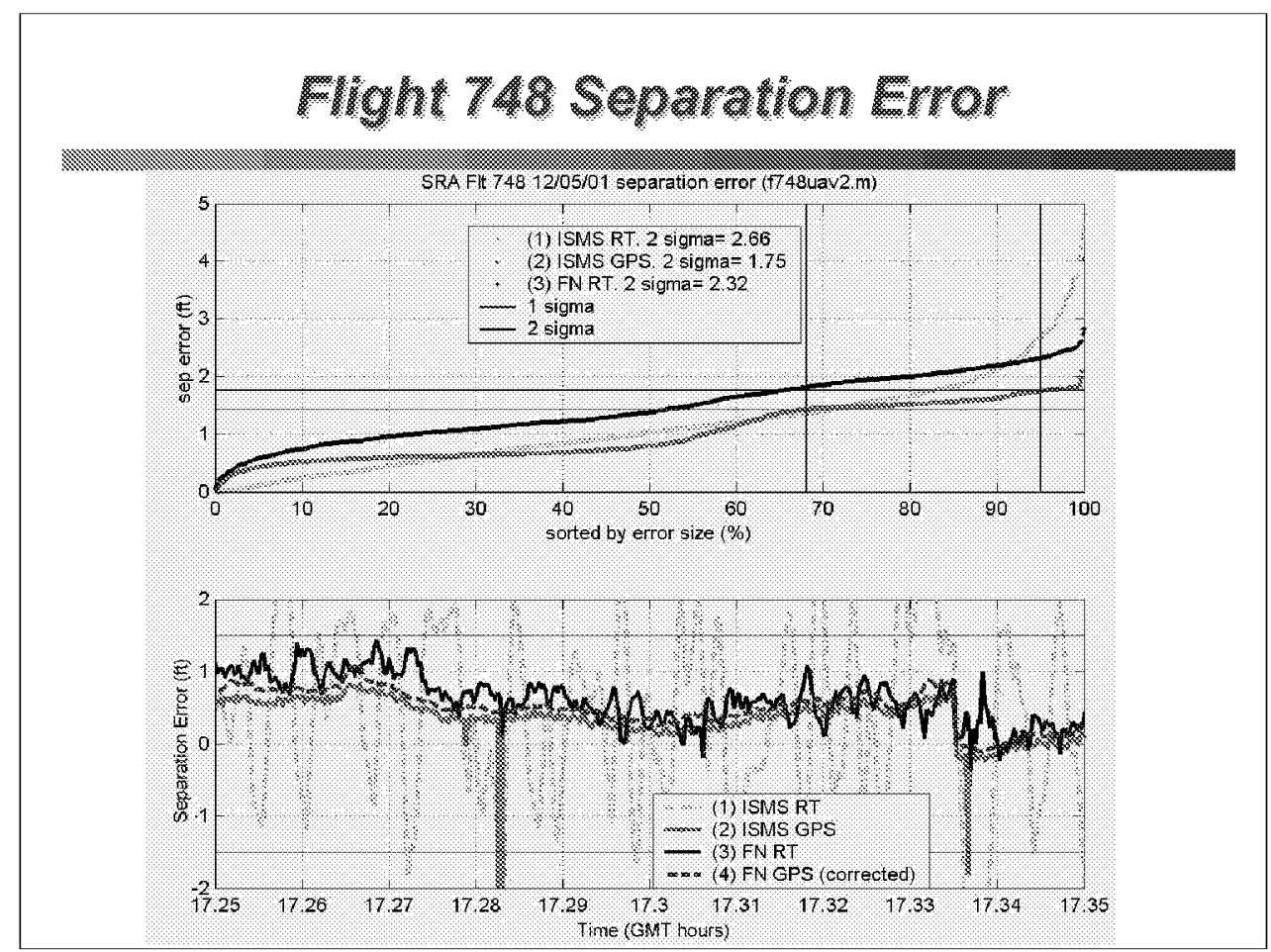

Flight 748 was a flight to demonstrate fuel savings while flying in the vortex. As the relative separation between the two aircraft was held constant throughout most of the flight, this represents the best case in terms of latency issues being minimized and probably has the best accuracy in GPS position reporting.

Note the scale change from previous separation error slides. The scale of the bottom plot was expanded from $\pm 5 \mathrm{ft}$ to $\pm 2 \mathrm{ft}$ because all of the data had better accuracy.

2-sigma errors of 1.8 to $2.7 \mathrm{ft}$ cover the range for all of the methods, although the ISMS swings are more pronounced than the other lines, as usual.

This illustrates that if the formation flight is fixed, and the heading and altitude of the formation remains largely unchanged, there is very little difference in the accuracy reported regardless of the technique used. 


\section{Flight Data Separation Error Sigma}

- Mlustration of sigma crror values in both 1 SWS and FN computations. One might ushing FrS is inchuded.

- Illustrates average error and variation between flights

- 2 to $4 \mathrm{ft} 2$-sigma (95 percent)

- 0.5 to $1.5 \mathrm{ft} 1$-sigma (68 percent)

- Flight data and sigma values

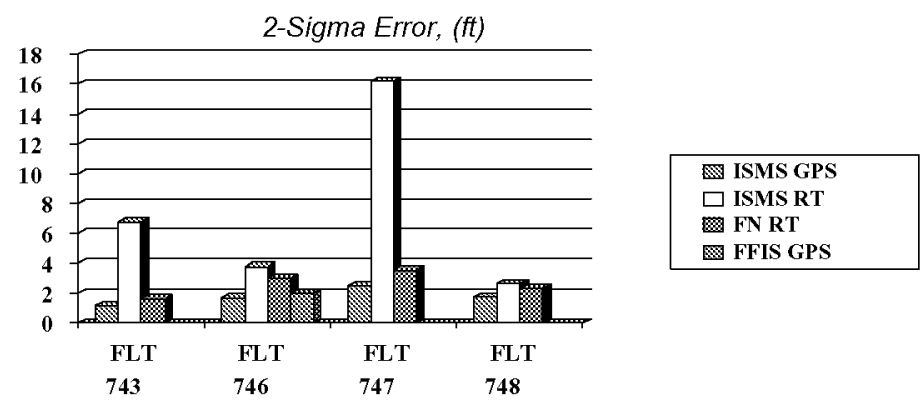

This slide summarizes the 2-sigma error data from the previous four separation error slides.

Remember that data known to be bad as a result of GPS satellite set mismatch, significant telemetry dropouts, aircraft separation out of relative range, or carrier phase not locked (in the case of the FFIS) were removed from the data set before the 1- and 2-sigma error values were calculated. This allowed the basic techniques to be compared on a more level playing field.

What is noteworthy is that a simple position differencing between the two aircraft (ISMS GPS) yields a 2-sigma error of about $2 \mathrm{ft}$ or less.

The ISMS RT can yield a significantly higher error, but with the exception of flight 747 is still within three to seven ft. The flight 7472 -sigma value is so much higher because of more significant accelerations and decelerations between the two aircraft.

The FN RT technique has an error close to the ISMS GPS. As the ISMS GPS represents the best case (no latency), this is a further indication that the extrapolation technique used in FN RT works well.

Only one flight of the FFIS GPS error was plotted, which shows about the same accuracy as the ISMS GPS. However, the FFIS system is still under development and these errors are expected to drop further. 


\section{Error Sources}

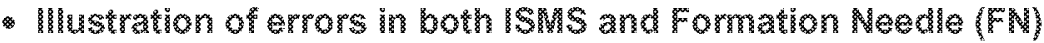
computations and description of what the 'glinchas' ard whe 10:

- GPS Satellite set difference

- GPS Satellite set change

- course/altitude/acceleration change extrapolation error

- out of separation range

- PC Modem telemetry loss

- Data from Inghts $743,744,746,747,748$ (whole Hights)

Next is a discussion about error sources observed during lab and flight testing, with some plots that illustrate their effect. A previous slide illustrated the acceleration and deceleration effect on the error reported by ISMS RT. 


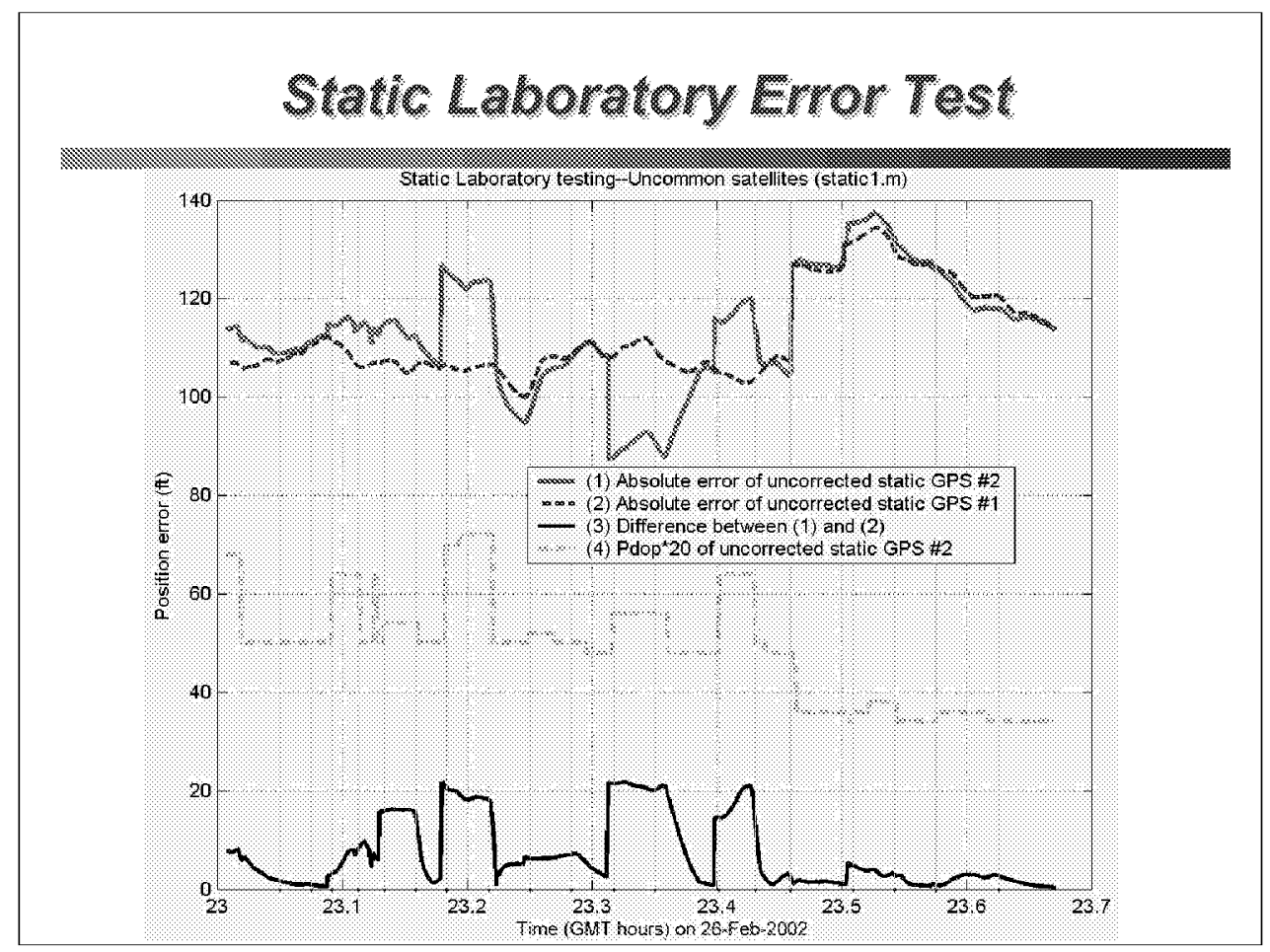

This plot contains some GPS data acquired in the laboratory to develop a static baseline for the effect of uncommon satellites and satellite transitions on the accuracy of separation data.

The setup was two Z-12 ${ }^{\mathrm{TM}}$ GPS receivers connected to one GPS antenna that had a surveyed position. One receiver (GPS no. 2) then had selected GPS satellites masked off and back on again over the space of about 0.7 hours. Data was collected by various means.

The graph shows the absolute position error of GPS no. 2, line (1) and the absolute position error of GPS no. 1, line (2).

The absolute position error of both is around 100 to $140 \mathrm{ft}$. However, the absolute value of the difference between those two lines is line (3) at the bottom, which is no greater than about $22 \mathrm{ft}$ at any time. The magnitude of the error is largely a function of the satellite geometry--as indicated by the position dilution of precision (PDOP) of line (4). The higher the PDOP, the worse the geometry of the satellite constellation.

At time 23.45, a new satellite was acquired by both receivers, which improved the geometry, but a greater absolute error was observed in both positions for other reasons. However, since both receivers tracked each other (subject to the same error sources), the difference between them reduced considerably.

The vertical gray lines indicate times when satellite masks were either turned on or off (some of which might not even be used by the receivers).

The conclusions from this test are:

- The relative position error between receivers is considerably less than the absolute error of each receiver position.

- Relative error with uncommon satellite changes yield a relative error of up to about $22 \mathrm{ft}$. Common satellites yield relative errors of around 2 or $3 \mathrm{ft}$--as seen in the flight data. 


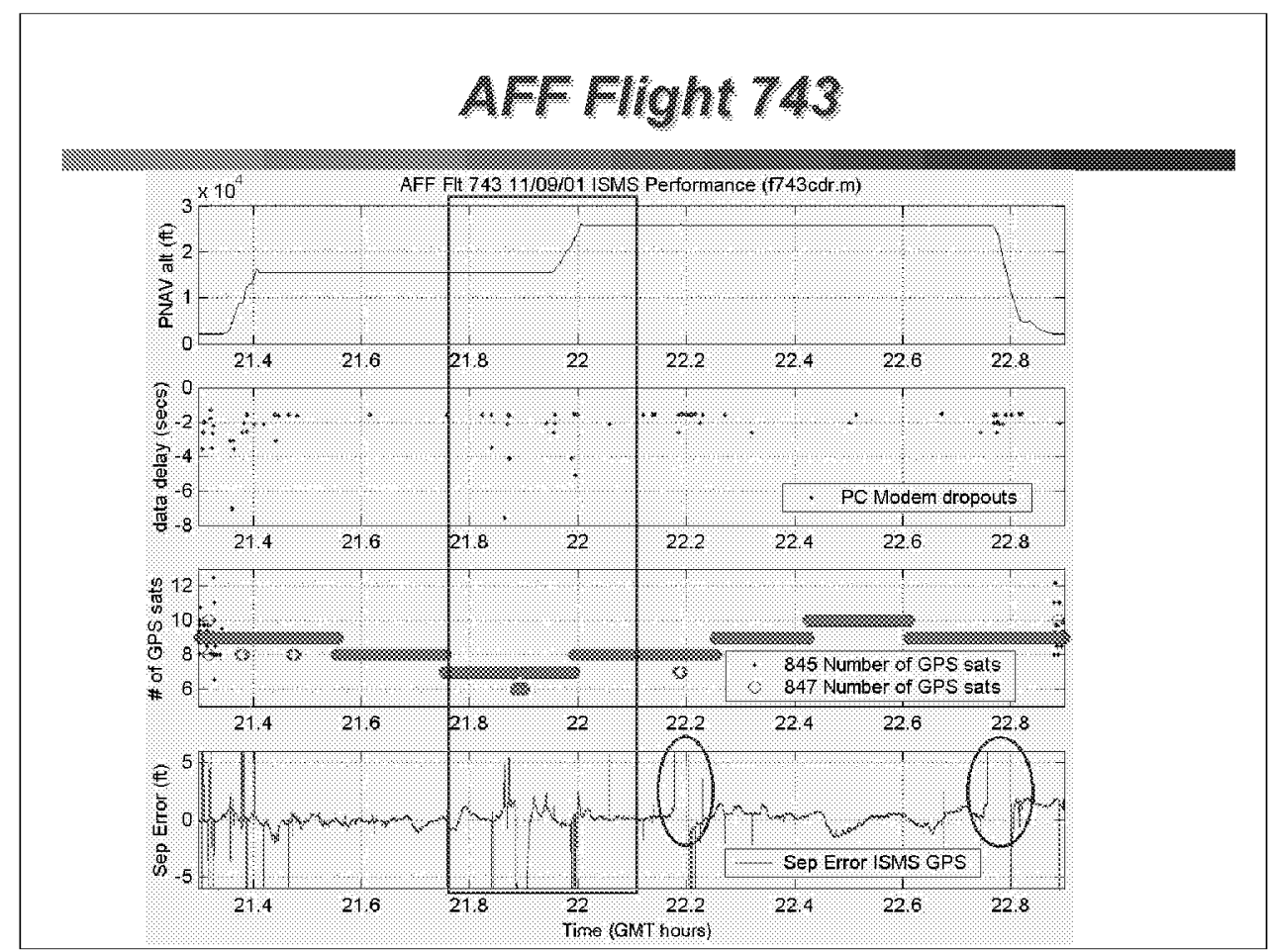

This collection of four plots shows the altitude profile for flight 743, PC radio modem dropouts, GPS satellite changes for both aircraft (as an indicator of uncommon satellites), and separation error for the ISMS GPS.

This collection of plots covers the whole of flight 743 .

$\mathrm{PC}$ radio modem dropouts of the second plot were measured by subtracting real time from the GPS time tagged with the data. If the receiving modem receives nothing or receives data that is not 100-percent correctable, that packet is ignored. The more consecutive packets that are missed, the more negative the indication on the plot is.

Circles show aircraft separation out of range ( $>3276.7 \mathrm{ft}$ ), so the separation error number is automatically driven to a value of 3276.7 (16-bit full positive with a $0.1 \mathrm{ft}$ resolution) in order to easily identify its condition. This is among the data removed from the sigma calculations.

The errors at the beginning were a result of taxi telemetry communication problems and were also removed from the sigma calculations.

The area enclosed by the rectangle is expanded on the next slide. 


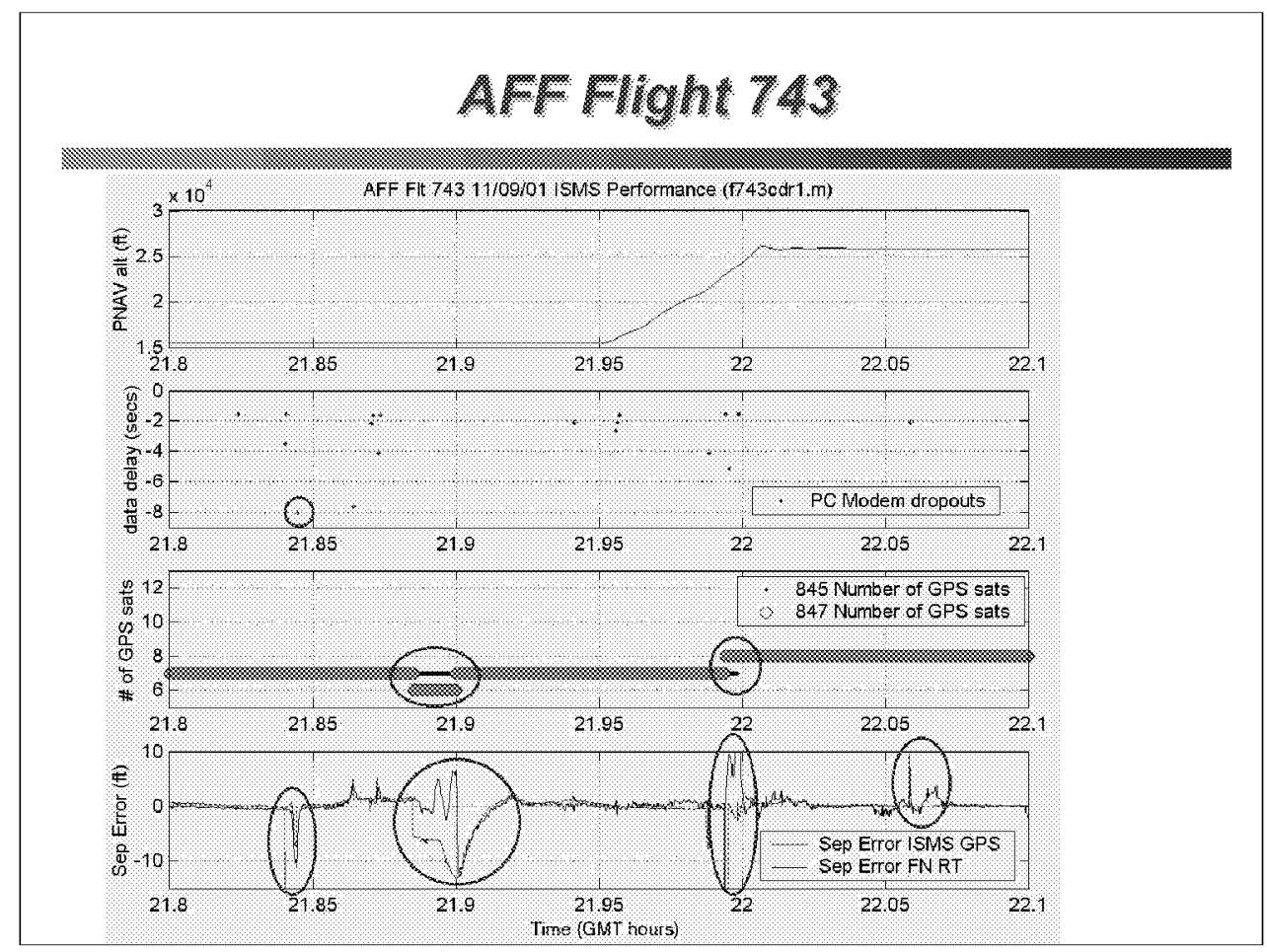

This segment of flight 743 , an enlarged view of the area enclosed by the rectangle on the previous slide, has circled error events.

The GPS satellite plot shows two times where the satellites between the aircraft did not match. During these periods the FN calculation freezes, so the indication of error here is only accurate if the two aircraft remain at the same relative separation as before the freeze. From the bottom plot 'Sep Error FN RT' line, it is seen that the error indication wanders around depending on what changes in relative aircraft separation have occurred while the FN calculation was frozen.

The ISMS GPS calculation continues during a GPS satellite mismatch, however, and around time 21.9 it is seen to drop toward an error of about $13 \mathrm{ft}$. This is consistent with the lab data. When the satellites match again, the error rises exponentially back toward zero. It takes typically about one minute to settle back to pre-mismatched values as shown here.

At time 22, another GPS satellite mismatch occurs, but this time the ISMS GPS error does not increase as much. This is because of two factors: the satellite geometry was not impacted as much so the weighting of the satellite positioning was not as significant as before; and the mismatch was not as long as the first one.

Near time 21.85, a circle highlights two types of errors. The first is a glitch caused by a PC radio modem dropout. ISMS takes the glitch because its data is not filtered. The FN RT (black) plot 'motors through' this as a result of its digital filtering. The second dip is also caused by a PC radio modem dropout, but a longer one. The point above it on the 'data delay' plot is a -8 , which means a data loss of 8 seconds. This time, even the filtering of the FN couldn't overcome it. 


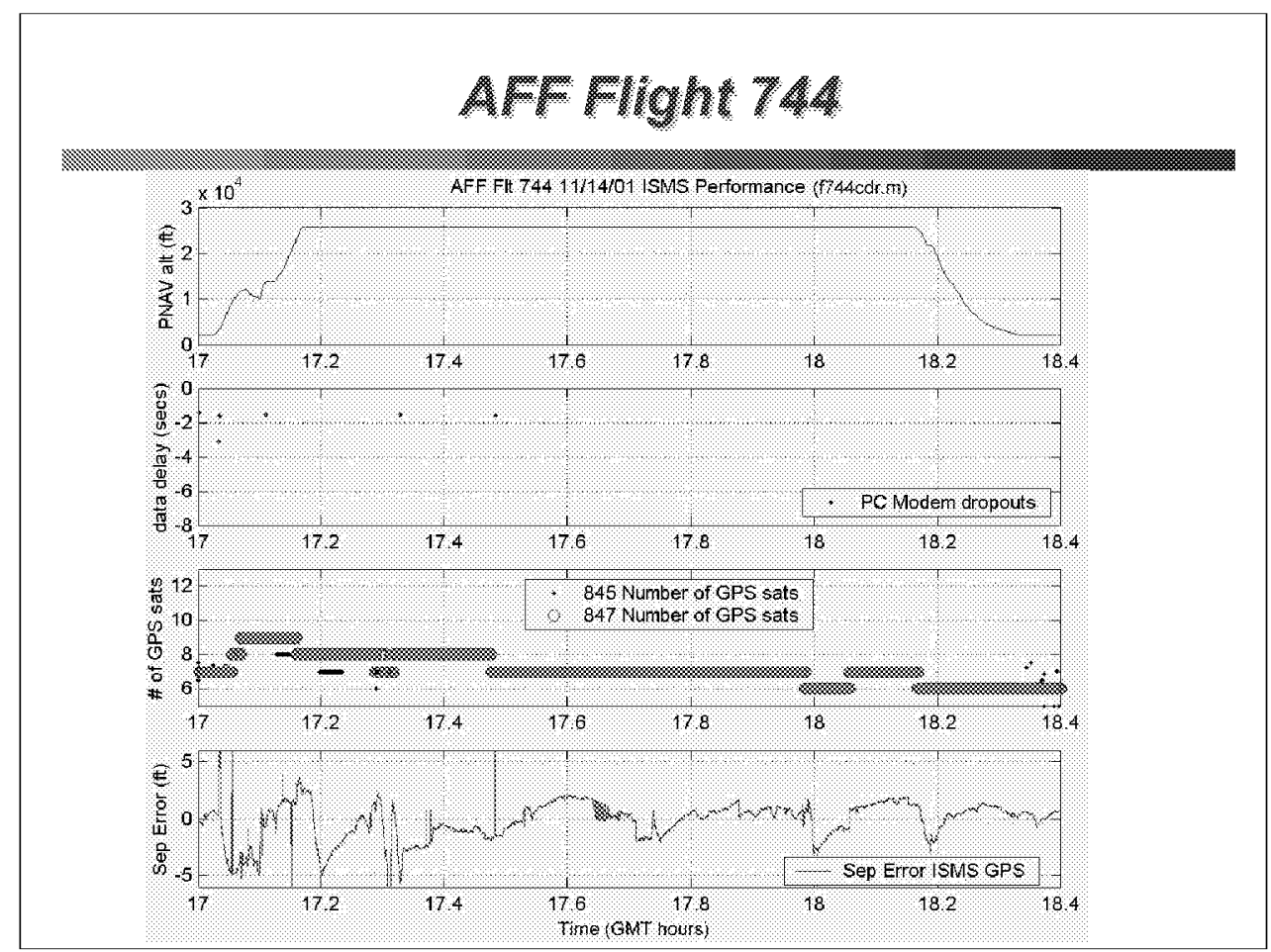

This and succeeding slides illustrate the effects of the separation error, PC radio modem dropouts, and GPS satellite changes for several flights.

Notice that this flight (744) had very few radio modem dropouts, and its satellite mismatches and changes had little effect on the ISMS GPS accuracy. This is probably caused by mismatched satellites being low on the horizon and not being large contributors to the PDOP. 


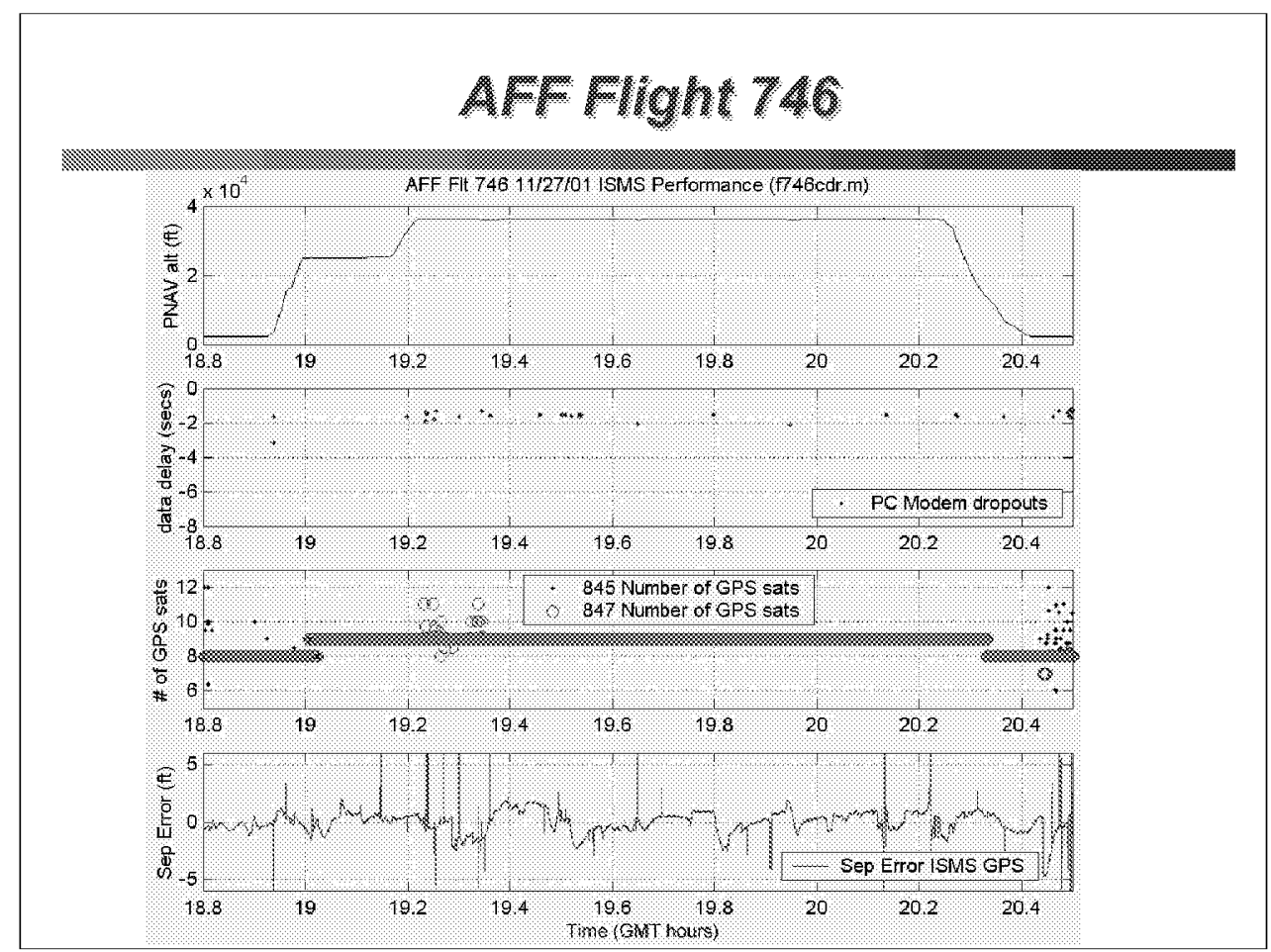

This flight 746 slide shows more typical flight data. 


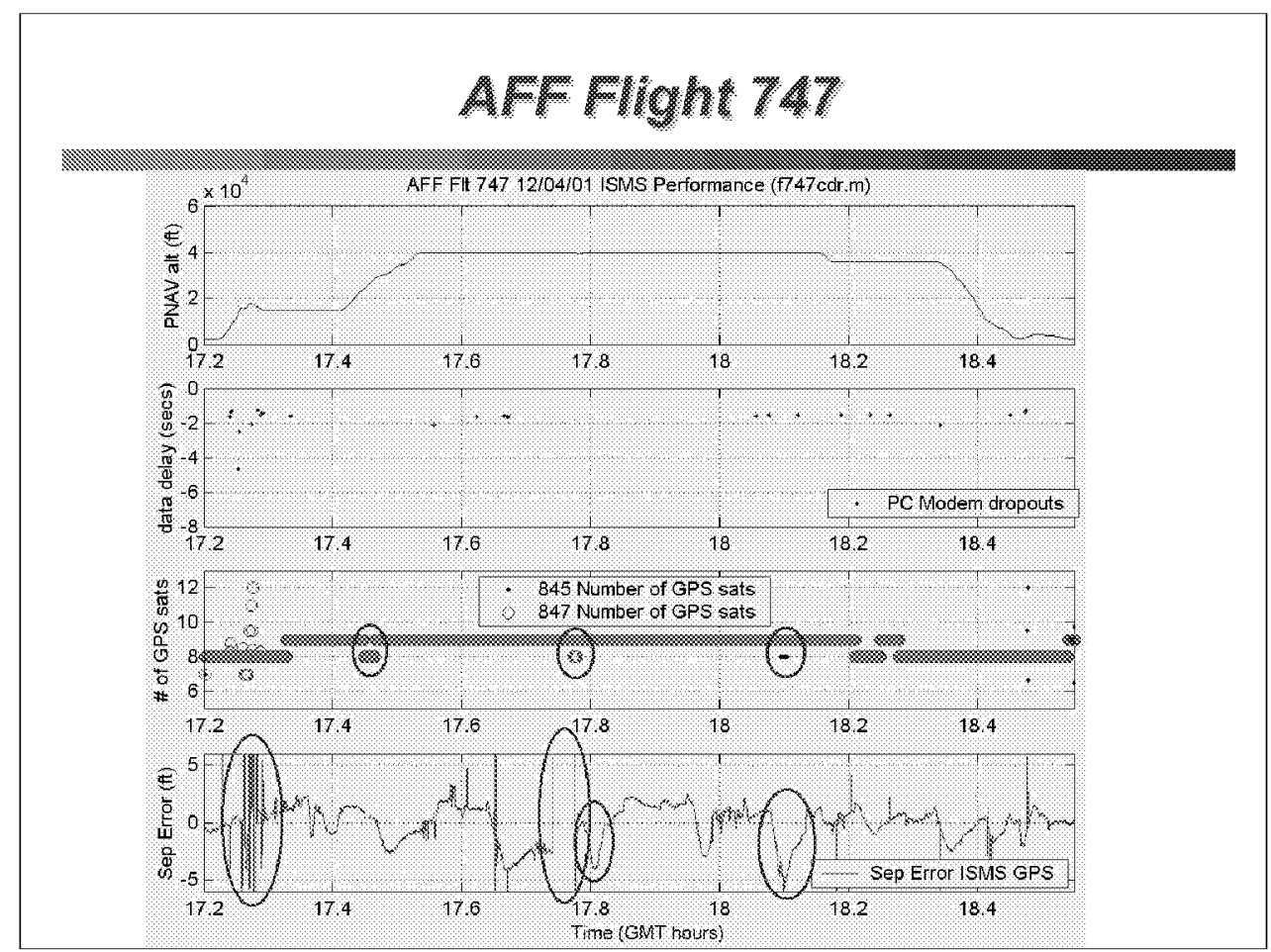

The circles on the third plot of flight 747 (number of GPS satellites) indicate where there was a satellite mismatch between the Z-12 ${ }^{\text {TM }}$ GPS receivers on the two aircraft. At time 17.45, there was little reaction on the fourth plot (Separation Error). Following the 'out of range' condition circled at time 17.75, there is another satellite mismatch, and the separation error is larger than the first time. At time 18.1, the satellite mismatch results in an even larger error (about $5 \mathrm{ft}$ ).

The 'noise' circled at time 17.3 is the result of telemetry dropouts during takeoff. 


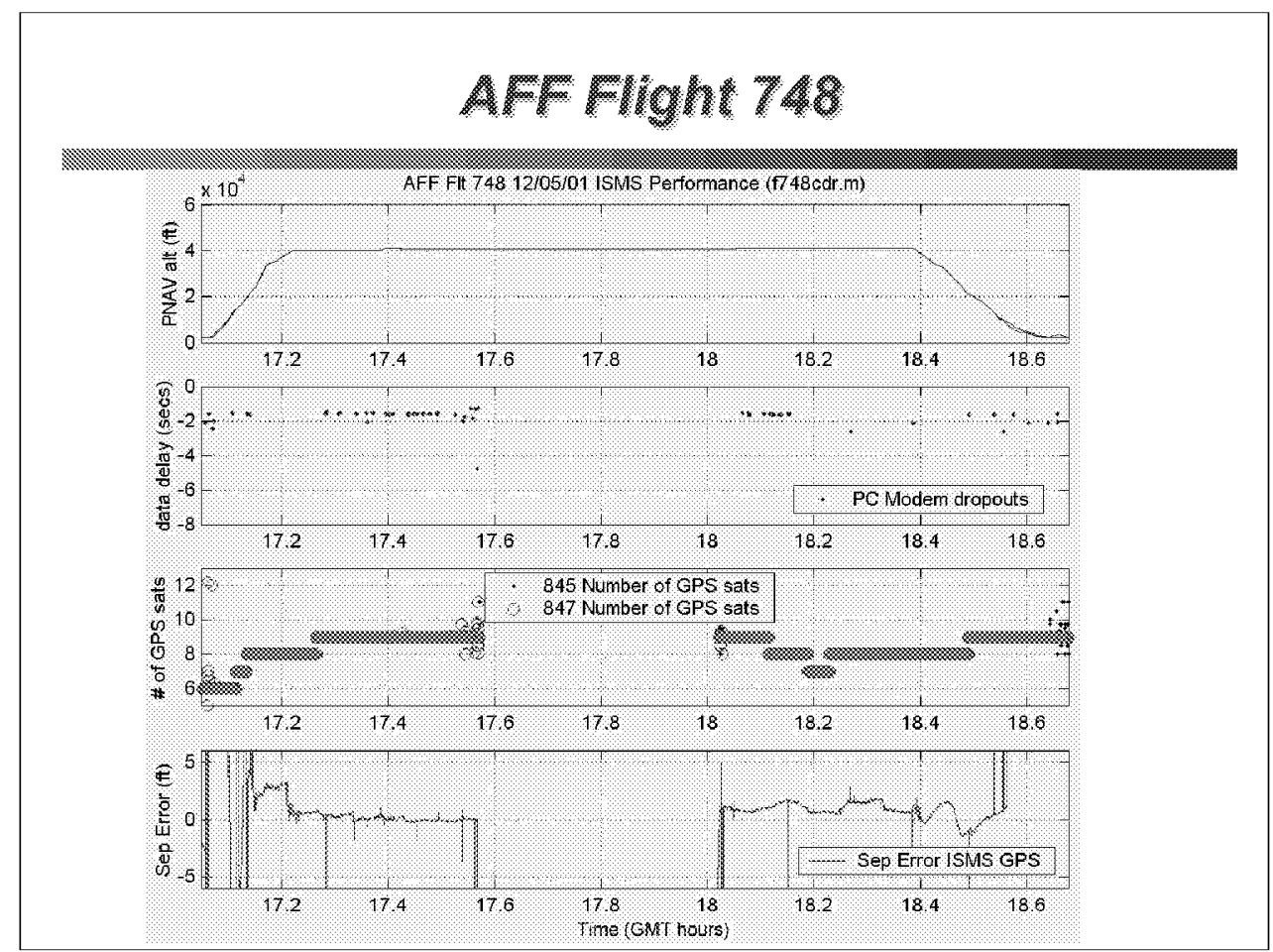

There is a gap in the middle of the flight 748 plots here because the aircraft flew out of range. It was about 200 miles away from the telemetry ground station when the slant range was too great for tracking. Except for the $\mathrm{Z}-12^{\mathrm{TM}}$ GPS receiver and FFIS internal memories, no other data was recorded onboard the trailing aircraft. 


\section{Observations}

- ISMS \& Mubject to common error sources which tend to cance.

- ISWS FN Whn not function well

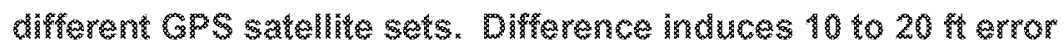
instead of usual 2.5 ť.

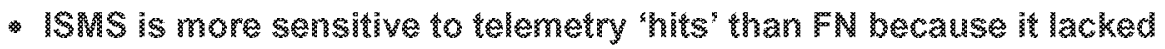
a digutulturer.

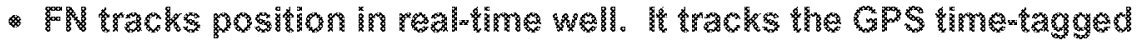
data. The extrapolation works.

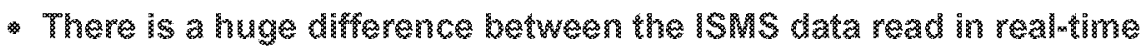
vs. that tagged wh chs time.

- FFis is not sufficienty developer to be robust. There is enough flight data to amine its potental. Hs computed ralative position comparas well with postulyht corrected data.

The ISMS, which is a GPS-only solution, would not work well as a pilot aid to positioning. Its update rate (2 $\mathrm{Hz}$ ) is too slow and its data is delayed by one-half second.

The FN system, which is also a GPS-only solution, does work well as a pilot positioning aid in real time. Its 'virtual' update rate is $10 \mathrm{~Hz}$, and the transport delay of one-half second was largely removed by extrapolation. However, it was not designed to work 'well' during maneuvering. This was not a problem as all of the flight test points were in reasonably stable conditions (no large, rapid changes in altitude or course). Errors increase as a result of extrapolation error while maneuvering. The extrapolation strategy would have to be reworked for the maneuvering case.

The ISMS RT and ISMS GPS yield very different error results. Integrated over time, the errors are generally much closer to each other. This is because they are both derived from the same data in the same data acquisition process.

The FFIS was in a development stage and was not mature by the end of the flight test.

Alignment of data with the appropriate time is critical for the error analysis. There were seven time 'sources' that had to be correlated (leading aircraft recorded GPS, trailing aircraft recorded GPS, leading aircraft PCM real time, trailing aircraft PCM real time, trailing aircraft GPS time, leading aircraft GPS time as received by trailing aircraft, and FFIS recorded GPS time). ISMS RT and ISMS GPS are a graphic illustration of the different results that can be obtained by plotting the same data against different time sources. 


\section{Conclusions}

- ISMS

- good for proximity alert separation measurement

- not good for pilot-in-the-loop control

- error is nominally 2 to $6 \mathrm{ft} 2$-sigma in real time

- simple solution

- Formation needles (FN)

- good for pilot-in-the-loop control in relatively stable flight

- error is nominally $2.5 \mathrm{ft} 2$-sigma

- OK if occasional GPS mismatches can be tolerated

- Fris

- best approach for taking real-time advantage of both GPS and IMU

- good for providing for GPS loss

- higher dynamics of IMU

- best and potentially most reliable for control system integration

- Verification of system is difficult

The ISMS and FN were designed for different purposes and each performed its task well. ISMS is the simplest approach. FN was better suited for pilot-in-the-loop control, as expected. Both yielded acceptable errors for many piloting tasks. The virtual update rate of $10 \mathrm{~Hz}$ (based on $2 \mathrm{~Hz}$ actual data) was adequate for the pilot positioning task.

FFIS has more potential to be a robust solution that is insensitive to:

- GPS satellite set difference between aircraft

- GPS satellite set change

- Course and altitude change extrapolation error

- Out of separation range.

The FFIS would also be sensitive to communication loss between the two aircraft, but less so than with the ISMS or FN because of IMU data inclusion. However, much more development is required to realize its potential. 
Public reporting burden for this collection of information is estimated to average 1 hour per response, including the time for reviewing instructions, searching existing data sources, gathering and maintaining the data needed, and completing and reviewing the collection of information. Send comments regarding this burden estimate or any other aspect of this collection of information, including suggestions for reducing this burden, to Washington Headquarters Services, Directorate for Information Operations and Reports, 1215 Jefferson Davis Highway, Suite 1204 , Arlington, VA 22202-4302, and to the Office of Management and Budget, Paperwork Reduction Project (0704-0188), Washington, DC 20503.

\begin{tabular}{|l|l|l|}
\hline 1. AGENCY USE ONLY (Leave blank) & $\begin{array}{l}\text { 2. REPORT DATE } \\
\text { June } 2002\end{array}$ & $\begin{array}{l}\text { 3. REPORT TYPE AND DATES COVERED } \\
\text { Technical Memorandum }\end{array}$ \\
\hline
\end{tabular}

4. TITLE AND SUBTITLE 5. FUNDING NUMBERS

Comparison of Relative Navigation Solutions Applied Between Two Aircraft 6. AUTHOR(S)

WU 7063500 E8 $2800 \mathrm{AFF}$

Glenn Bever, Peter Urschel, and Curtis E. Hanson

7. PERFORMING ORGANIZATION NAME(S) AND ADDRESS(ES)

8. PERFORMING ORGANIZATION

NASA Dryden Flight Research Center

P.O. Box 273

Edwards, California 93523-0273

National Aeronautics and Space Administration

Washington, DC 20546-0001

NASA/TM-2002-210728

11. SUPPLEMENTARY NOTES

Presented at the AIAA 1st Technical Conference and Workshop on Unmanned Aerospace Vehicles, Systems, Technologies and Operations, May 20-23, 2002, Portsmouth, Virginia.

\begin{tabular}{l|l} 
12a. DISTRIBUTION/AVAILABILITY STATEMENT & 12b. DISTRIBUTION CODE \\
Unclassified-Unlimited & \\
Subject Category 04 & \\
This report is available at http:/www.dfrc.nasa.gov/DTRS/ &
\end{tabular}

13. ABSTRACT (Maximum 200 words)

Use of global positioning systems (GPS) for guidance and control functions is of increasing interest to the aviation industry. Many levels of solutions exist, from the relatively simple to highly complex integrated systems. This presentation examines three different GPS approaches to determining the relative separation between two aircraft. It presents flight test data showing the errors in each of the three approaches, tradeoffs in selecting from these three approaches, and the simplifying assumptions made for implementing applications that may reduce the requirements and therefore the cost of using them.

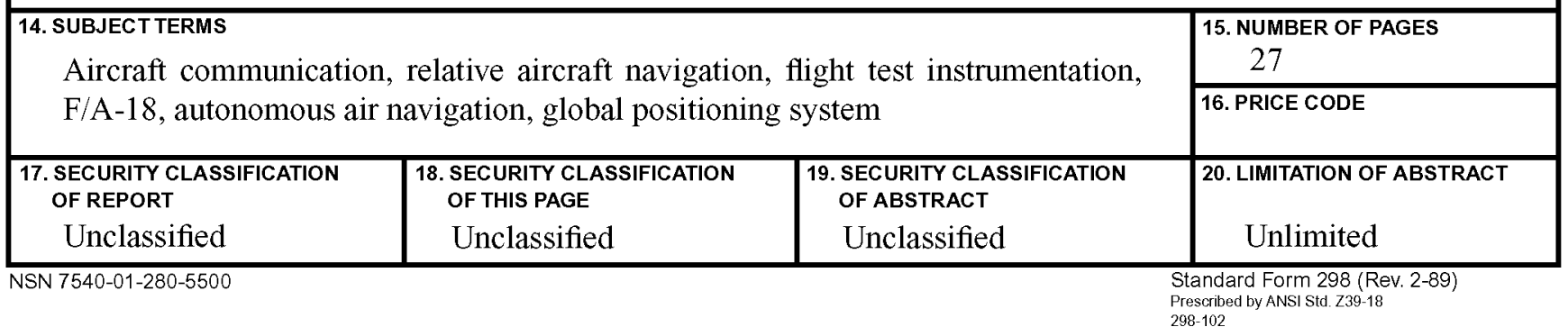

